\title{
Solução Analítica do Problema de Impacto em Barras Viscoelásticas
}

\author{
Carlos Friedrich Loeffler Pedro V. M. Pereira Abraão L. C. Frossard \\ Universidade Federal do Espirito Santo, Departamento de Engenharia Mecânica \\ 29075-910, Vitoria, ES \\ E-mail: carlosloeffler@bol.com.br \\ E-mail: eng.pedrompereira@gmail.com \\ E-mail: abraaocaldas@hotmail.com
}

\begin{abstract}
RESUMO
Normalmente nos projetos rotineiros de engenharia costuma-se trabalhar com materiais que apresentam um comportamento mecânico constitutivo independente do tempo. No entanto, com a evolução dos materiais e a inclusão de polímeros dentre os materiais usados na construção em engenharia, tornou-se importante incluir o efeito da dependência do tempo no comportamento destes materiais quando submetidos a uma dada carga. Entre os muitos efeitos, encontram-se os efeitos viscosos de relaxação, fluência e deslizamento. Por outro lado, muitos problemas da engenharia moderna envolvem carregamentos dinâmicos, de modo que os modelos matemáticos resultantes são complexos por acoplar simultaneamente o caráter dependente do tempo da resposta e do material constitutivo. Para resolvê-los, métodos numéricos discretos têm sido desenvolvidos, com formulações específicas para a sua abordagem, o que requer soluções analíticas para melhor aferição da qualidade de seus resultados. Com o intuito de gerar tais soluções de referência, este trabalho apresenta a solução analítica de uma barra visco elástica sujeita a uma carga de impacto, através do tradicional Método de Separação de Variáveis.
\end{abstract}

Para construção do modelo matemático considera-se um sólido de Maxwell adaptado [4], em que uma mola e amortecedor em série (Modelo de Maxwell) estão em paralelo com uma mola auxiliar, conforme apresenta a figura 1 :

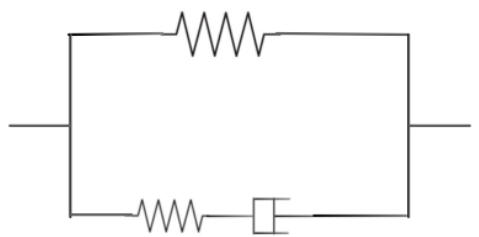

Figura 1: Modelo de Maxwell adaptado

Neste caso, a equação que relaciona tensão e deformação é dada por [3]:

$\frac{\partial \sigma}{\partial t}=E^{*} \frac{\partial^{2} u}{\partial x \partial t}-\frac{\sigma}{\tau}$

Eq. 1

Onde E* é o módulo de elasticidade resultante das molas, $\sigma$ é a tensão inicialmente aplicadas, $\boldsymbol{\tau}$ é o tempo de relaxamento do material e u representa o deslocamento ao longo da direção x. A segunda lei de Newton neste caso é expressa por [1]:

$\rho \frac{\partial^{2} u}{\partial t^{2}}=\frac{\partial \sigma}{\partial x}$

Eq. 2

Compondo estas duas equações, a equação diferencial parcial que governa a propagação de ondas axiais é representada por: 
$\rho \frac{\partial^{3} u}{\partial t^{3}}-E^{*} \frac{\partial^{3} u}{\partial x^{2} \partial t}+\frac{\rho}{\tau} \frac{\partial^{2} u}{\partial t^{2}}=0$

Eq. 3

Ressalta-se que neste modelo o comportamento elástico e dissipativo estão em paralelo. A solução desta equação pode ser obtida considerando-se que:

$\rho \frac{\partial^{2} u}{\partial t^{2}}-E^{*} \frac{\partial^{2} u}{\partial x^{2}}+\frac{\rho}{\tau} \frac{\partial u}{\partial t}=\gamma$

O valor de gama é retirado da análise das condições de segunda derivada no tempo e depende das propriedades viscoelásticas do material.

A solução é obtida através do Método de Separação de Variáveis [2] pode-se resolver a equação anterior considerando-se que a constante apresentada no lado direito da igualdade comporta-se como uma ação de domínio. Admitindo que o sistema consista de uma barra engastada numa extremidade, a solução pelo citado método fornece:

$$
\begin{aligned}
\gamma(x, t)=\frac{\gamma}{2} x^{2} & -\gamma l x \\
& +e^{\frac{-\rho t}{\tau}}\left[\left(\sum_{n=1,5,9}^{\infty}\left(\frac{16 \gamma l^{2}}{n^{3} \pi^{3}}\right) \operatorname{sen}\left(\frac{n \pi x}{2 l}\right) \cos \left(\omega_{n} t\right)\right)\right. \\
& \left.+\left(\sum_{n=3,7,11}^{\infty}\left(\frac{16 \gamma l^{2}}{n^{3} \pi^{3}}\right) \operatorname{sen}\left(\frac{n \pi x}{2 l}\right) \cos \left(\omega_{n} t\right)\right)\right]
\end{aligned}
$$

A representação gráfica dos deslocamentos ao longo do tempo é similar ao de um problema de impacto amortecido.

Palavras-chave: Materiais visco elásticos, Método de Separação de Variáveis, Soluções Analíticas de Referência, Modelo de Maxwell adaptado.

\section{Referências}

[1] R. M. Caddell, "Deformation and Fracture of Solids", Prentice-Hall, 1980.

[2]R. C. Juvinall, "Engineering Considerations of Stress, Strain and Strength", McGraw-hill, 1967.

[3]H. Kolsky, "Stress Waves in Solids", Dover, 1963.

[4]E. Kreiszig, Matematica Superior, vol 3, Livros Técnicos e Científicos Editora, Rio de Janeiro, 1976. 